\title{
RAG-1 mutations that affect the target specificity of $V(D) J$ recombination: a possible direct role of RAG-1 in site recognition
}

\author{
Moshe J. Sadofsky, Joanne E. Hesse, Dik C. van Gent, and Martin Gellert \\ Laboratory of Molecular Biology, National Institute of Diabetes and Digestive and Kidney Diseases (NIDDK), Bethesda \\ Maryland 20892 USA
}

The RAG-1 protein plays an essential role in $V(D) J$ recombination, but its exact function has not yet been defined. Here we report that a particular mutation in RAG-1 affects recombination by altering the specificity of target sequence usage. Recombination mediated by wild-type RAG-1 is tolerant of a wide range of coding sequences adjacent to the recombination signal. With the mutant RAG-1, recombination is much more demanding; efficient recombination is only found when particular dinucleotides are adjacent to the signal sequence heptamer. The mutant is also more sensitive than wild-type RAG-1 to certain alterations within the signal sequence. We suggest that the RAG-1 protein may interact physically with the target DNA at the coding-signal sequence border.

[Key Words: V(D)/ recombination; RAG-1; mutagenesis; plasmid library]

Received June 15, 1995; revised version accepted July 13, 1995.

$V(D) /$ recombination is the site-specific DNA rearrangement that assembles the gene segments encoding the antigen receptors of the immune system. The target site for recombination is marked by the presence of specific signal sequences adjacent to each coding segment. These signal sequences, composed of conserved heptamer and nonamer motifs separated by a spacer of length 12 or 23 $\mathrm{bp}$, are the only essential recognition elements. The coding sequences flanking the signals are highly variable, and are found in recombined junctions without great selectivity, although some preferences have been described (Boubnov et al. 1993; Gerstein and Lieber 1993; Ezekiel et al. 1995).

The products of the RAG-1 and RAG-2 genes (Schatz et al. 1989; Oettinger et al. 1990) are the only lymphoidspecific factors required for $V(D) I$ recombination, and their expression is sufficient to activate $V(D) /$ recombination in non-lymphoid cells. Recent studies in a cellfree system demonstrated that RAG-1, and probably RAG-2, acts directly in the initial cleavage step of $V(D) I$ recombination (van Gent et al. 1995). The exact activities of these factors, however, are still not known and, in particular, it has not been determined whether either protein plays a role in the recognition of signal sequences. Indeed, it has not been possible to assign either protein a distinct role in the reaction, as no alterations in target DNA are detected unless both proteins are present (Mombaerts et al. 1992; Shinkai et al. 1992; Schlissel et al. 1993).

One approach to uncovering specific activities attrib- utable to RAG-1 is a mutational analysis of the protein. We and others (Sadofsky et al. 1993; Silver et al. 1993) recently found that a large amino-terminal segment of the RAG-1 gene is expendable for the formation of signal junctions in extrachromosomal substrates. A core region, consisting of residues 384-1008, still supports a high level of recombination. In general, local mutations in the amino-terminal region allow full activity regardless of the plasmid substrate used to test them, whereas most mutations in the core region abolish activity (Sadofsky et al. 1993).

To determine a more precise function for RAG-1 in $V(D) /$ recombination, we searched for mutations in RAG-1 that make recombination hypersensitive to the exact sequence of substrates. We have identified a RAG-1 mutant (D32) that exhibits a strong preference for recombining particular substrates. The significant difference between favored and unusable substrates maps to the coding sequence adjacent to the heptamer of the signal. This coding flank preference of the RAG-1 D32 mutant has been further defined with the help of a randomized library of coding ends. The D32 mutant is also disproportionately sensitive to changes in some bases of the heptamer. We suggest that RAG-1 may make direct contact with the DNA at the coding-heptamer border.

\section{Results}

To learn more about the function of RAG-1 in $V(D) I$ recombination, we previously introduced a series of $\mathrm{mu}$ - 
tations into the core region of RAG-1 required for recombination (amino acids 384-1008). Recombination activity was assayed by cotransfection of each mutant with a RAG-2 expression plasmid and a substrate into fibroblasts. In the course of analyzing the properties of these mutations, we observed that one mutant (D32) of this truncated protein has the striking property of recombining one of our standard deletion substrates (pJH200) almost as well as wild-type RAG-1 while recombining a second deletion substrate (pJH289) 1000-fold less efficiently. This observation was puzzling, because these two substrates are very similar. The sequences of the recombination signals in the two plasmids are substantially identical, and they both generate signal joints upon recombination. Recombination frequencies measured with the two plasmids and wild-type RAG-1 are usually quite high (Table 1).

To understand what sequences contributed to this substrate specificity, we systematically exchanged fragments of the two plasmids. We found that the relevant differences were in the first few nucleotides of coding sequence immediately adjacent to the heptamer of the signal sequence. In particular, RAG-1 D32, was sensitive to changes within the $5 \mathrm{bp}$ of coding sequence nearest to either the 12- or 23-signal. Changes in any other parts of the plasmids had no effect (data not shown).

Plasmids that generated a coding joint, either by deletion or inversion, were similarly affected (Table 1). We compared recombination activity of D32 and its wildtype counterpart on four coding joint-forming substrates (Fig. 1) that differed only in the five nucleotides adjacent

Table 1. Recombination activities of RAG-1 variants on four test substrates

\begin{tabular}{lcccc}
\hline & \multicolumn{4}{c}{ Recombination (\%) } \\
\cline { 2 - 5 } RAG-1 & $\begin{array}{c}\text { pJH200 } \\
\text { signal joint }\end{array}$ & $\begin{array}{c}\text { pJH289 } \\
\text { signal joint }\end{array}$ & $\begin{array}{c}\text { pJH290 } \\
\text { coding joint }\end{array}$ & $\begin{array}{c}\text { pJH299 } \\
\text { inversion }\end{array}$ \\
\hline $1-1040$ & 2.3 & 9.8 & 2.2 & 0.1 \\
$384-1008$ & 3.2 & 31.3 & 6.2 & 0.2 \\
D32 & 0.9 & 0.001 & 0.002 & $<.00003$ \\
D39 & 0.7 & N.D. & 0.002 & N.D. \\
\hline
\end{tabular}

Full-length RAG-1 (residues 1-1040) is encoded in plasmid pJH548. The core residues 384-1008 are encoded in plasmid pMS127B. In the D32 variant of the truncated RAG-1 (in pMS132), six residues of the core region are replaced with two others resulting in an additional net deletion of four. The D39 variant restores the amino-terminal 383 residues absent in D32. Substrates pJH200 and pJH289 test the formation of signal junctions. Substrate pJH290 tests formation of coding junctions and pJH299 forms both junctions in an inversional rearrangement. Plasmids pJH289, pJH290, and pJH299 carry the same coding sequences adjacent to their recombination signals. The recombinants reported here reflect conventional rearrangements confirmed by hybridization and exclude alternative forms such as hybrid junctions. Experiments with low recombination were repeated several times, screening at least $1,000,000$ plasmids for each datum. Each D32 result represents between 5 and 10 independent repetitions. (N.D.) not determined.

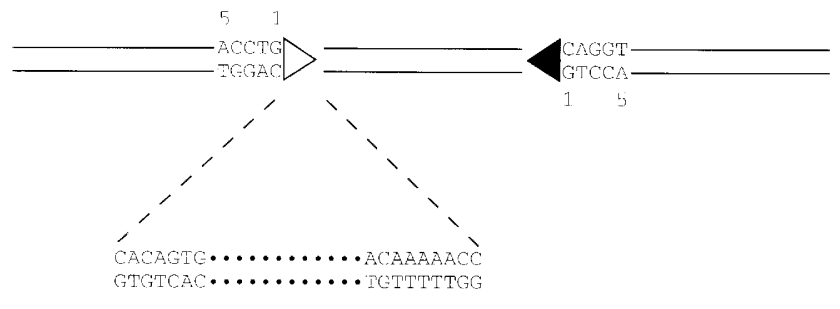

Figure 1. A substrate (pMS319) that forms a coding joint after recombination. The portion containing the recombination signals is shown. $(\Delta)$ The 12-signal; $(\Delta)$ the 23-signal. The sequence ACCTG is found 5' to each recombination signal. Coding nucleotides at the first and fifth positions preceding the heptamer are indicated. In this orientation, the signals and the DNA between them are deleted during recombination, and the remaining plasmid DNA retains a coding joint. (Below) The consensus sequence of the 12-signal, with heptamer, spacer (dots), and nonamer.

to one or both signal sequences (Table 2). A substrate with the coding sequence ACCTG / read 5' to $3^{\prime}$ toward each signal) is recombined at comparable levels by D32 and wild type RAG-1. In contrast, substrates with either GATTC or TCGAC flanking one signal /with the preferred flank of ACCTG at the other) were poor substrates for RAG-1 D32. Their recombination frequencies were 90- to 140-fold lower than with wild-type RAG-1. When both of these "bad" flanks are combined the effect is compounded, and recombination directed by D32 is reduced $\sim 1000$-fold, down to the background level of the assay.

To explore further the coding sequence preferences of RAG-1 D32, we made a library of plasmid substrates in which the 5 coding nucleotides flanking the 12-signal were randomized while a "good" coding flank was kept constant at the 23-signal. Recombined plasmids recovered following transfection should thus reflect the sequence preference of the mutant. Because coding ends are often altered during the joining process, it was necessary to build the library in such a way as to preserve an exact copy of the intact coding flank. This was accomplished by incorporation of two complementary pentanucleotides in the library plasmid, one at the signal border to be used in the recombination event, with the other at an internal position where it would remain unchanged and available for analysis (Fig. 2). The initial distribution of random bases in the pentanucleotide was assessed by the sequencing of 64 members of the initial library pool. While guanine was under-represented in the input library by twofold $(\mathrm{A}=33 \%, \mathrm{C}=24 \%, \mathrm{G}=11 \%$, and $\mathrm{T}=31 \%$ ), no further individual or dinucleotide bias was seen at any particular position within the pentamer.

The substrate library was cotransfected into fibroblasts along with expression vectors for RAG-2 and either wild-type RAG-1 or D32. Among the many recombinants generated from two independent transfections, clones were chosen at random for sequence analysis. In addition to the internal copy of each pentanucleotide, the coding junction of each clone was sequenced to as- 
Table 2. Recombination of coding joint-forming substrates by wild-type RAG-1 and D32

\begin{tabular}{|c|c|c|c|c|c|}
\hline \multicolumn{2}{|c|}{ Coding sequence } & \multicolumn{2}{|c|}{ Recombination (\%) } & \multirow{2}{*}{$\begin{array}{l}\text { Ratio: D32 } \\
\text { to wild type }\end{array}$} & \multirow{2}{*}{$\begin{array}{l}\text { Substrate } \\
\text { name }\end{array}$} \\
\hline $5^{\prime}$ to 12 -signal & $5^{\prime}$ to 23 -signal & RAG-1 D32 & wild-type RAG-1 & & \\
\hline 54321 & 54321 & & & & \\
\hline ACCTGcac & ACCTGcac & 3.0 & 3.9 & 0.77 & pMS319 \\
\hline ACCTGcac & GATCCcac & 0.02 & 2.8 & 0.007 & pMS325 \\
\hline TCGACcac & ACCTGcac & 0.03 & 2.8 & 0.011 & pMS326 \\
\hline TCGACcac & GATTCcac & 0.002 & 2.2 & 0.0009 & pJH290 \\
\hline
\end{tabular}

The three nucleotides of the heptamer adjacent to the coding sequence (cac) are shown in lowercase, and the proximal coding sequence in uppercase letters. The wild-type RAG-1 used here is the full-length protein. The pJH290 data are from Table 1. Coding-end bases are numbered by position from the heptamer.

sure that only independent events were counted. When occasional identical clones were identified from a single transfection, they were counted as only one event, given the high probability that they were siblings. The original coding sequence (as reflected by the untouched internal complementary pentanucleotide) was determined for 51

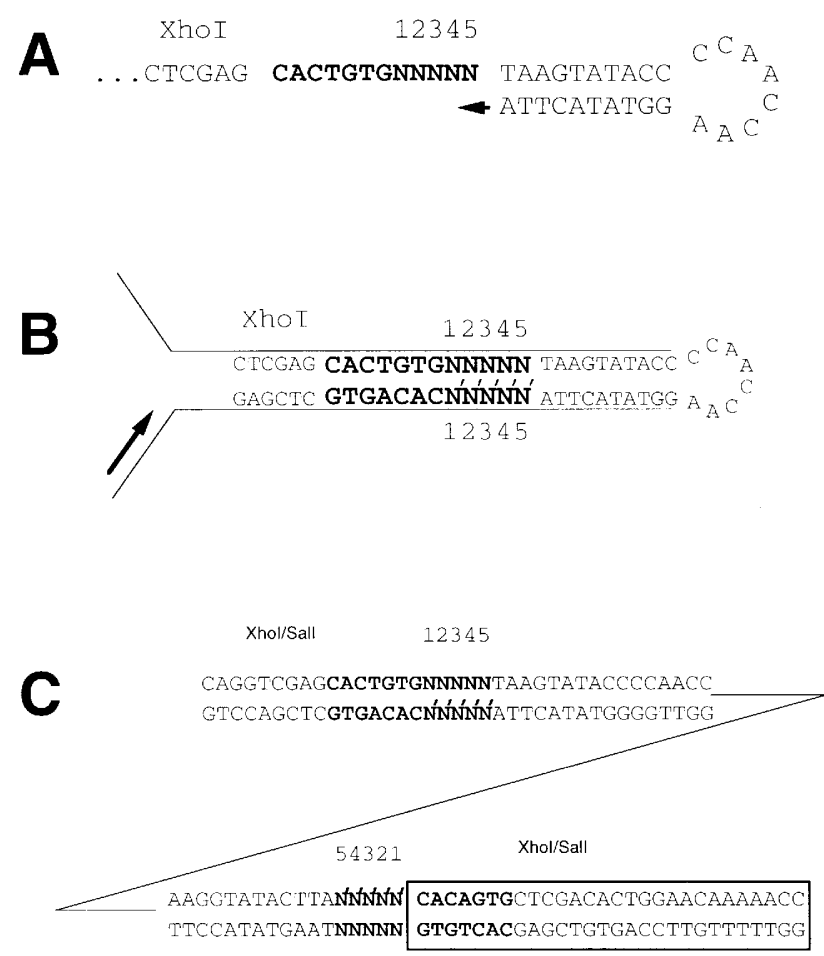

Figure 2. Construction of the random coding-end library. $(A)$ Initial oligonucleotide spontaneously forms a snap-back structure that can prime itself for subsequent enzymatic extension to a fully double stranded product. $(B)$ The product of primer extension is cleaved with $\mathrm{XhoI}$ and ligated to a fishtail-linker, which results in the structure shown. Primer extension from the arrow around the hairpin produces a fully double-stranded product with two complementary copies of the random nucleotides. $(C)$ The product of $B$ is cleaved with $X h o I$ and ligated into a SalI site in a vector designed to complete the signal sequence (boxed) by addition of a nonamer to only one side. One of the two possible orientations is shown. independent recombinants generated by wild-type RAG-1 and 105 from RAG-1 D32. When the distribution of nucleotides at individual positions was tabulated and compared, no significant deviation from the original distribution was found for either set of recombinants. When the distribution of adjacent dinucleotides was analyzed, however, a great preference for particular dinucleotides adjacent to the heptamer was revealed for RAG-1 D32 (Fig. 3), whereas wild-type RAG-1 showed no obvious preference. In particular, the coding ends most over-represented with RAG-1 D32 (TA and TG) were not overrepresented in the wild-type case.

Contingency table and $\chi^{2}$ analysis of the data obtained with RAG-1 D32 indicate that the distribution of nucleotides does not differ significantly from random among the three positions distal to the heptamer, whereas the dinucleotide distribution proximal to the heptamer is skewed from random at the 0.001 confidence level. If the additional three nucleotides have an influence, it is small relative to the effect of the dinucleotide.

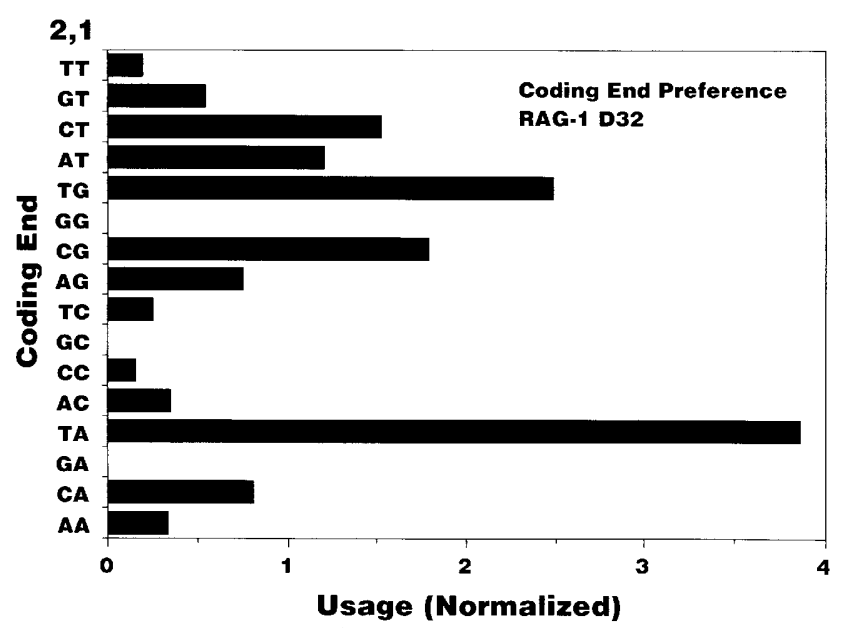

Figure 3. Coding-end preference of RAG-1 D32. The terminal dinucleotide of coding sequence of recombinants generated from the library of randomized coding ends was analyzed. Coding-end bases are numbered by position from the heptamer. The usage of each dinucleotide was normalized to correct for the bias in input distribution. A value of 1 would represent the expected frequency in the absence of selection. 
The library screen thus reflected a sequence preference of the D32 mutant for the dinucleotide adjacent to the coding flank. To determine more exactly the effect on recombination frequency of the preferred and under-represented sequences, individual substrates with these sequences flanking the 12-signal were made and tested with D32 or wild-type RAG-1. In some plasmids, the nucleotide in the third position was also changed, and in others, the flanks of both signals were modified.

Recombination of these substrates is consistent with the results obtained from the library screen (Table 3 ). Here also the coding-end nucleotides TA and TG are recombined with D32 almost as well as with wild-type RAG-1. Other coding-end dinucleotides such as TC, CC, GG, and GA once again yield much lower recombination with D32. Each substrate exhibits a similar high level of recombination with wild-type RAG-1. [Note that the plasmid that has the lowest recombination with wildtype RAG-1 carries the TTT-coding-end DNA shown by others to suppress recombination /Gerstein and Lieber 1993; Ezekiel et al. 1995; however, the mutant to wild type ratio is high.] Certain pairs of substrates illustrate that replacement of nucleotides at the third position from the heptamer border has little influence on the activity ratio. For example, ACCTG and ACTTG both have high ratios of D32 to wild type, and A $\bar{C}$ TAA and ACAAA have lower but similar ratios. These experiments confirm that RAG-1 D32 is sensitive to coding sequence, because a 100 -fold change in recombination can be produced by alteration of the dinucleotide adjacent to one heptamer. Even larger effects are apparent if both coding ends are altered simultaneously, as in pMS374 (Table 3).

While we were alerted to the substrate selectivity of the RAG-1 D32 mutant by its preference for certain cod- ing flanks, we were also interested in the possibility that it would show an altered or greater sensitivity to changes in the signal sequence. Recombination with wild-type RAG-1 is known to be greatly reduced by mutations in any of the first three positions of the heptamer (nearest the coding sequencel, but tolerates mutations in the other four bases of the heptamer and most positions of the nonamer (Hesse et al. 1989). We therefore tested recombination on plasmids with an altered heptamer or nonamer in the 12-signal (Table 4). A coding end sequence favored by D32 (ACCTG) was preserved at both signals. As expected, alteration of the third base of the heptamer led to very low recombination with both wildtype RAG-1 and D32. However, changes in positions 4 or 5 of the heptamer gave strikingly depressed recombination with D32 compared to wild-type RAG-1. Recombination with D32 was also hypersensitive to simultaneous mutation of positions 5,6 , and 7 or position 7 alone. On the other hand, nonamer mutations in the 12-signal sequence led to parallel reductions in recombination with both wild-type RAG-1 and D32 (Table 4).

Is the behavior of the D32 mutation unique? This mutation was made in the background of a large deletion that removed residues 1-383 of RAG-1. A first question was whether the amino-terminal deletion contributed to its properties. When the same amino acid changes were made in RAG-1 with the full-length amino terminus (mutant D39), however, the recombination behavior paralleled that of D32 (Table 1). We also made a number of other mutations in the region of the protein near the D32 site, between amino acids 570 and 640 . As summarized in Figure 4, some of these mutants were active with all substrates, whereas others were totally inactive. The target selectivity found in D32 is evidently not mimicked by nearby mutations. Even at the site of the D32 muta-

Table 3. Recombination of substrates that differ at coding-end sequences with full-length-wild-type RAG-1 (pIH548) and RAG-1 D32

\begin{tabular}{|c|c|c|c|c|c|}
\hline \multicolumn{2}{|c|}{ Coding sequence } & \multicolumn{2}{|c|}{ Recombination $(\%)$} & \multirow{2}{*}{$\begin{array}{l}\text { Ratio: D32 } \\
\text { to wild type }\end{array}$} & \multirow{2}{*}{$\begin{array}{l}\text { Substrate } \\
\text { name }\end{array}$} \\
\hline $5^{\prime}$ to 12 -signal & $5^{\prime}$ to 23 -signal & RAG-1 D32 & wild-type RAG-1 & & \\
\hline 54321 & 54321 & & & & \\
\hline ACCTGcac & ACCTGcac & 3.0 & 3.9 & 0.77 & pMS319 \\
\hline TCGACcac & ACCTGcac & 0.03 & 2.8 & 0.01 & pMS326 \\
\hline ACTTAcac & ACTTGcac & 0.8 & 3.4 & 0.23 & pMS334 \\
\hline ACTTGcac & ACCTGcac & 0.7 & 1.7 & 0.41 & pMS335 \\
\hline ACTAAcac & ACCTGcac & 0.4 & 4.1 & 0.10 & pMS370 \\
\hline ACTTTcac & ACCTGcac & 0.3 & 0.7 & 0.43 & pMS371 \\
\hline ACTTCcac & ACCTGcac & 0.1 & 4.2 & 0.02 & pMS373 \\
\hline ACGCTcac & ACCTGcac & 0.08 & 1.1 & 0.07 & pMS336 \\
\hline ACACCcac & ACCTGcac & 0.02 & 2.6 & 0.01 & pMS337 \\
\hline ACAGAcac & ACCTGcac & 0.05 & 2.0 & 0.02 & pMS338 \\
\hline ACAAAcac & ACCTGcac & 0.1 & 1.6 & 0.06 & pMS339 \\
\hline ACGGGcac & ACCTGcac & 0.03 & 2.1 & 0.01 & pMS340 \\
\hline ACTTAcac & ACTTAcac & 0.7 & 2.2 & 0.32 & pMS341 \\
\hline ACTGGcac & ACTGGcac & 0.002 & 2.4 & 0.001 & pMS374 \\
\hline
\end{tabular}

In each of these substrates, the signals are arranged to form coding junctions upon recombination. Each result reflects from two to seven independent experiments. The three nucleotides of the heptamer adjacent to the coding sequence (cac) are shown in lowercase and the proximal coding sequence in uppercase letters. Coding-end bases are numbered by position from the heptamer. 
Table 4. Effect of nonconsensus 12-signal heptamers and nonamers on recombination with RAG-1 D32

\begin{tabular}{llclll}
\hline & \multicolumn{2}{l}{ Recombination $(\%)$} & & Ratio: \\
\cline { 2 - 4 } $\begin{array}{l}\text { 12-Signal } \\
\text { heptamer }\end{array}$ & $\begin{array}{c}\text { RAG-1 } \\
\text { D32 }\end{array}$ & $\begin{array}{c}\text { wild-type } \\
\text { RAG-1 }\end{array}$ & $\begin{array}{l}\text { D32 to } \\
\text { wild-type }\end{array}$ & $\begin{array}{l}\text { Substrate } \\
\text { name }\end{array}$ \\
\hline cacagtg & 0.8 & 2.2 & 0.36 & pMS319 \\
caGagtg & 0.01 & $<0.002$ & NA & pDVG30 \\
cacTgtg & 0.004 & 1.3 & 0.003 & pDVG29 \\
cacaAtg & 0.04 & 1.7 & 0.024 & pDVG28.2 \\
cacagtA & 0.09 & 1.0 & 0.09 & pDVG31 \\
cacaACA & 0.03 & 2.8 & 0.01 & pDVG32 \\
12-Signal & & & & \\
nonamer & & & & \\
acaaaaacc & 0.7 & 2.4 & 0.29 & pDVG33 \\
acaaGaacc & 0.4 & 1.6 & 0.25 & pDVG35 \\
acaaaaGcc & 0.07 & 0.2 & 0.35 & pDVG36 \\
acaCGaacc & 0.1 & 0.3 & 0.33 & pDVG37 \\
\hline
\end{tabular}

The plasmids all carry the sequence ACCTG 5 ' to both signals. Within each set, plasmids vary at the capitalized nucleotides. The heptamer or nonamer is shown in the orientation of the signal in Fig. 1. Recombination takes place at the left side of the heptamer as drawn. Wild-type RAG-1 in these experiments is the truncated version (residues 384 1008). Similar results were obtained with the full-length RAG-1 (not shown). (N.A.) not applicable.

tion, the net deletion of 4 residues in D32 is compatible with function, but deletion of 2 residues at the same site (D47) results in an inactive protein.

Although no other mutation in this set displayed the modified substrate specificity of D32, a recently detected RAG-1 variant has an amino acid substitution at position 609 (C. Roman, pers. comm.) and appears, in our hands, to have a behavior related to that of D32 (data not shown). This position is covered by the deletion in D32, supporting the idea that this site may have a special role in DNA recognition.

\section{Discussion}

Recent studies with a cell-free system strongly suggest that RAG-1 protein acts directly in the site-specific cleavage that initiates $V(D) /$ recombination /van Gent et al. 1995). However, the precise role and functions of RAG-1 remain unclear. Here we have identified a mutation in RAG-1 that greatly alters the protein's ability to stimulate recombination when the substrate DNA contains certain coding sequences and renders recombination hypersensitive to some changes in the signal sequence heptamer. The formation of coding joints or signal joints is affected similarly, suggesting that the mutation influences an early event in recombination, most probably the recognition of target sites.

The simplest interpretation of these results is that RAG-1 binds directly to the DNA at the coding end and heptamer. But because we are detecting only complete recombination, it is also possible that the $\mathrm{D} 32$ mutation affects a later sequence-dependent stage in the reaction. For example, RAG-1 D32 might be able to bind to all signal-coding borders, but only participate in later chemical steps if the right coding flank is present. Alternatively, it is possible that the specificity is mediated through other factors. Thus, an additional DNA-binding protein needed for the reaction may normally interact with RAG-1, but the D32 mutation might weaken this interaction such that only the highest affinity DNA sites allow recombination. In any of these interpretations, it is not clear why there is recognition of the nonconserved sequence flanking the signals, and this point requires further study.

The hypersensitivity of RAG-1 D32 to DNA sequence around the signal heptamer does not seem to be related to a more general inhibition of $V(D) /$ recombination observed with wild-type RAG-1 when certain homopolymeric DNA tracts are used as coding flanks /Gerstein and Lieber 1993, Boubnov et al. 1993; Ezekiel et al. 1995). In those cases, changes of one or two bases in one coding flank resulted in rather small effects on recombination, and large effects resulted only from the use of long tracts on both flanks. With our substrates as well, local base changes at one coding end had relatively small effects on recombination mediated by wild-type RAG-1 (Table 3). The variation observed with D32, however, was much greater. We conclude that the $\mathrm{D} 32$ mutation in RAG-1 imposes more stringent sequence requirements for efficient recombination.

It should be noted that the present experiments deal only with extrachromosomal substrates. It is not yet known whether the same selectivity among coding flank sequences will extend to integrated substrates or to the antigen receptor loci of mice expressing D32 in place of wild-type RAG-1. Such experiments are now being designed.

An alternative explanation for our results might be

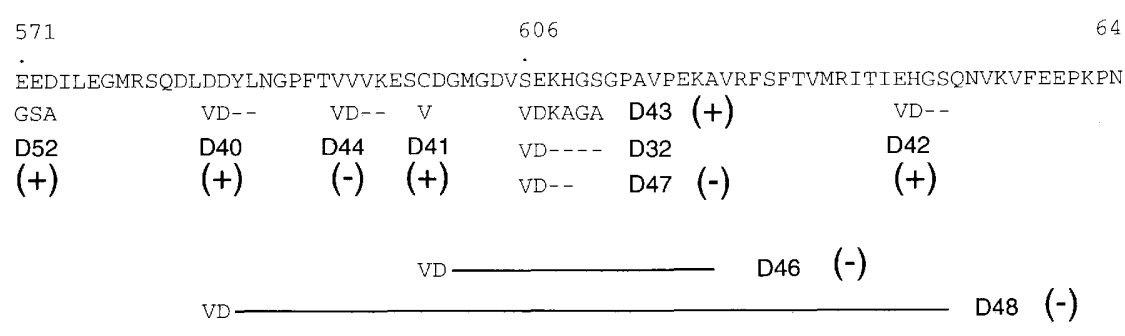

Figure 4. The amino acid sequence of mouse RAG-1 (Schatz et al. 1989) from residues 571 to 648 is shown, with the mutational changes below. Dashes represent deleted residues, and solid lines extended deletions. Residue 609 has been corrected (C. Roman, pers. comm.). Activity on recombination substrates is shown in parentheses. $1+\mid$ Mutants were active on both $\mathrm{pJH} 200$ and $\mathrm{pJH} 290 ;(-)$ mutants were inactive on both. D32 is discussed in the text. 
that D32 merely has reduced activity and that the target specificity of this mutant is inherently present in the normal protein, but can be seen only when the activity is too low to saturate the sites. This possibility is unlikely for several reasons. First, the D32 mutation does not alter RAG-1 protein level. When a B-cell line was transfected with a RAG-1 expression construct and the ${ }^{35} \mathrm{~S}$ labeled protein was immunoprecipitated (as described by Sadofsky et al. 1993), the steady-state levels of D32 and wild-type RAG-1 proteins were indistinguishable. Like the wild-type protein, the D32 protein was found to localize to the nucleus, implying also that it must be correctly folded (data not shown). Second, to reduce the intracellular level of protein, we tested a nonreplicative version of the plasmid expressing the wild-type RAG-1 core region but found no effect on substrate specificity (data not shown). Third, other RAG-1 constructs known to reduce the protein stability (those introducing point mutations in the Cys-His-rich region (Sadofsky et al. 1993; Silver et al. 1993) do not show any such change in specificity (data not shown). Fourth, in transfections with wild-type RAG-1, we have not been able to mimic the D32 coding end sequence preferences through manipulation of DNA dose or time course (data not shown).

In summary, the D32 mutation appears to change the recognition specificity of $V(D) /$ recombination. These results provide genetic evidence, complementary to biochernical evidence (van Gent et al. 1995), for a direct role of RAG-1 in the recombination reaction, and suggest that this role may be in sequence recognition.

\section{Materials and methods}

\section{Plasmids}

Full-length unaltered RAG-1 and RAG-2 are encoded in the expression plasmids pJH548 and 549 respectively (Sadofsky et al. 1993). The plasmids expressing the truncated active version of RAG-1 (pMS127B) and mutant D32 (pMS132) are also described in that paper. Each site-specific mutation introduced into RAG-1 in this report was made by PCR amplification of a seg. ment of DNA that was subsequently substituted for the wildtype sequence in that region in pMS127B. The desired changes were confirmed by DNA sequencing (Sequenase kit; U.S. Biochemical/Amersham). The mutations are described below. The number listed in the mutation description is that of the first altered residue in the wild-type sequence. The sequence listed before the number is replaced by that which follows. Extended deletions are listed as del A-B inclusive: D32, SEKHGS 606 VD; D40, DDYL 584 VD; D41, C 599 V; D42, EHGS 632 VD; D43, SEKHGS 606 VDKAGA; D44, VVK 594 D; D46, del 599-619 VD; D47, SEKH $606 \mathrm{VD}$; and D48, del 584-635 VD. The plasmid pMS139 encoding the D39 RAG-1 mutant was constructed by the swapping of the SphI-MluI fragment of pMS132 containing the deletion into the equivalent position of plasmid pMS119C (Sadofsky et al. 1993), thereby restoring the fulllength amino-terminal sequence.

Substrates pJH200 (Hesse et al. 1987), pJH289, pJH290 (Lieber et al. 1988), and pJH299 (Hesse et al. 1989) have been reported previously. New substrate plasmids were all constructed in the backbone of pJH299, first by removal of both signals and the intervening DNA by digestion with SalI and BamHI. Mutations were introduced by PCR with a variety of specific oligonucleotides and were confirmed by DNA sequencing. Details of individual plasmids are available upon request.

The extrachromosomal substrate assay was performed and chloramphenicol-resistant colonies were characterized further by hybridization with a battery of labeled probes as described previously (Sadofsky et al. 1994). Because recombinants expected to contain a signal joint can be probed for the creation of this junction, the screening of signal joint-forming substrates and inversion substrates is more stringent than of coding jointforming substrates. Consequently, the value of $0.002 \%$ represents the limit of sensitivity for this assay for pJH290 and other coding joint-forming substrates. Candidate recombinant coding joints derived from the library experiments were sequenced.

\section{Library construction}

The 54-base oligonucleotide MS189 was synthesized (ABI 380B): 5 '-TTAACTAACTCGAGCACTGTGNNNNNTAAGTATACCCCAACCAAGGTATACTTA-3', where $\mathrm{N}$ represents a mixture of all 4 bases. The $103^{\prime}$-most nucleotides can self anneal to the 10 nucleotides immediately $3^{\prime}$ to the cluster of N's, generating a hairpin structure (see Fig. 2). The 3' end serves as a primer for extension with DNA polymerase I, Klenow fragment (New England Biolabs). A ${ }^{32} \mathrm{P}$ tracer was incorporated during the strand extension. The resulting hairpin had a fully double stranded stem and was gel purified. This DNA was then cleaved with the restriction enzyme Xhol (New England Biolabs|, leaving a 4-nucleotide 5' single-stranded overhang. Two additional oligonucleotides were synthesized, MS190 (5'AAAAACTTAACTAAC-3', and MS191 5'-TCGAGTTAGTTAAGGCCACCACCGACCGC- $3^{\prime}$ ). The latter was phosphorylated (T4 polynucleotide kinase; New England Biolabs), and the two annealed to create a fishtail linker with the complementary overhang. This linker was ligated (T4 ligase; New England Biolabs) to the first product to create a 92-bp hairpin with nonannealed termini, and again gel purified. The primer MS192 $15^{\prime}$ GCGGTCGGTGGTGGC-3') was annealed to the $5^{\prime}$ singlestranded region and then extended at high temperature /Vent polymerase; New England Biolabs) by denaturing at $95^{\circ} \mathrm{C}$, rapid cooling to $80^{\circ} \mathrm{C}$, followed by slow cooling to $65^{\circ} \mathrm{C}$ over 30 min, to create a 102-bp double-stranded product with two internal XhoI sites. These sites were cleaved and the liberated internal fragment was ligated into the unique SalI site of plasmid pMS329. This plasmid, pMS329, was constructed with a nonamer (but no heptamer) near the SalI site. When the library fragment is inserted at this site, one cluster of N's and the adjacent heptamer is assembled into a complete 12-signal. The remaining complementary $\mathrm{N}^{\prime} \mathrm{s}$ and heptamer do not form a complete signal sequence and are situated $\sim 40$-bp upstream. The ligation creates two hybrid XhoI-SalI sites, which are resistant to subsequent restriction enzyme digestion, allowing counterselection against reclosed parental molecules containing no insert. The plasmid pool was then electrotransformed (Bio-Rad Gene Pulser) into electrocompetent Escherichia coli DH10B (Life Technologies). Approximately 5,000 independent colonies were obtained. A sample of these was characterized in detail and about $70 \%$ contained the desired structure. The remainder contained rearrangements and large deletions that would be eliminated by the constraints of the experiment (intact $\mathrm{T}$ antigen and polyoma origin for replication, CAT gene for the recombination assayl.

\section{Acknowledgments}

We thank Fraser McBlane, Dale Ramsden, and the other members of the Laboratory of Molecular Biology for discussion 
that was essential for the completion of this work. We also thank Christopher Roman for permission to cite unpublished observations.

The publication costs of this article were defrayed in part by payment of page charges. This article must therefore be hereby marked "advertisement" in accordance with 18 USC section 1734 solely to indicate this fact.

\section{References}

Boubnov, N.V., Z.P. Wills, and D.T. Weaver. 1993. V(D|J recombination coding junction formation without DNA homology: Processing of coding termini. Mol. Cell. Biol. 13: 69576968.

Ezekiel, U.R., P. Engler, D. Stern, and U. Storb. 1995. Asymmetric processing of coding ends and the effect of coding end nucleotide composition on $\mathrm{V}(\mathrm{D}) \mathrm{J}$ recombination. Immunity 2: 381-389.

Gerstein, R.M. and M.R. Lieber. 1993. Coding end sequence can markedly affect the initiation of $V(D) I$ recombination. Genes \& Dev. 7: 1459-1469.

Hesse, J.E., M.R. Lieber, M. Gellert, and K. Mizuuchi. 1987. Extrachromosomal DNA substrates in pre-B cells undergo inversion or deletion at immunoglobulin V-(D)-J joining signals. Cell 49: 775-783.

Hesse, J.E., M.R. Lieber, K. Mizuuchi, and M. Gellert. 1989. $V(D) I$ recombination: A functional definition of the joining signals. Genes \& Dev. 3: 1053-1061.

Lieber, M.R., J.E. Hesse, S. Lewis, G.C. Bosma, N. Rosenberg, K. Mizuuchi, M.J. Bosma, and M. Gellert. 1988. The defect in murine severe combined immune deficiency: Joining of signal sequences but not coding segments in $V(D) J$ recombination. Cell 55: 7-16.

Mombaerts, P., J. Iacomini, R.S. Johnson, K. Herrup, S. Tonegawa, and V.E. Papaioannou. 1992. RAG-1-deficient mice have no mature B and T lymphocytes. Cell 68: 869-877.

Oettinger, M.A., D.G. Schatz, C. Gorka, and D. Baltimore. 1990. RAG-1 and RAG-2, adjacent genes that synergistically activate V(D)J recombination. Science 248: 1517-1523.

Sadofsky, M.J., J.E. Hesse, and M. Gellert. 1994. Definition of a core region of RAG-2 that is functional in V(D)J recombination. Nucleic Acids Res. 22: 1805-1809.

Sadofsky, M.J., J.E. Hesse, J.F. McBlane, and M. Gellert. 1993. Expression and $\mathrm{V}(\mathrm{D}) \mathrm{J}$ recombination activity of mutated RAG-1 proteins. Nucleic Acids Res. 21: 5644-5650.

Schatz, D.G., M.A. Oettinger, and D. Baltimore. 1989. The V(D) recombination activating gene, RAG-1. Cell 59: 1035-1048.

Schlissel, M., A. Constantinescu, T. Morrow, M. Baxter, and A. Peng. 1993. Double-strand signal sequence breaks in $V(D) I$ recombination are blunt, 5'-phosphorylated, RAG-dependent, and cell-cycle regulated. Genes \& Dev. 7: 2520-2532.

Shinkai, Y., G. Rathbun, K.-P. Lam, E.M. Oltz, V. Stewart, M. Mendelsohn, J. Charron, M. Datta, F. Young, A.M. Stall, and F.W. Alt. 1992. RAG-2-deficient mice lack mature lymphocytes owing to inability to initiate V(D)J rearrangement. Cell 68: 855-867.

Silver, D.P., E. Spanopoulou, R.C. Mulligan, and D. Baltimore. 1993. Dispensable sequence motifs in the RAG-1 and RAG-2 genes for plasmid V(D)] recombination. Proc. Natl. Acad. Sci. 90: 6100-6104.

van Gent, D.C., J.F. McBlane, D.A. Ramsden, M.J. Sadofsky, J.E. Hesse, and M. Gellert. 1995. Initiation of V(D)J recombination in a cell-free system. Cell 81: 925-934. 


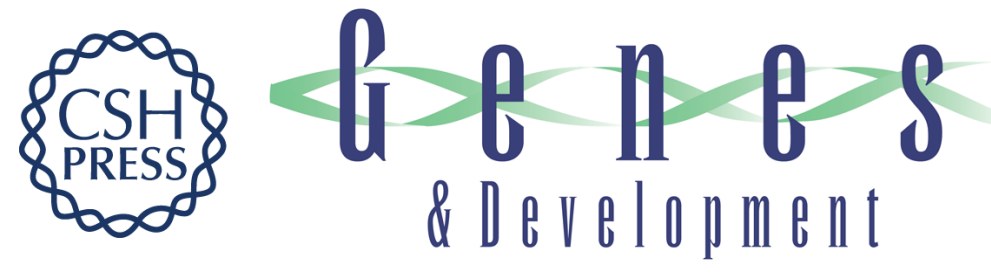

\section{RAG-1 mutations that affect the target specificity of $V(D) j$ recombination: a possible direct role of RAG-1 in site recognition.}

M J Sadofsky, J E Hesse, D C van Gent, et al.

Genes Dev. 1995, 9:

Access the most recent version at doi:10.1101/gad.9.17.2193

References This article cites 15 articles, 6 of which can be accessed free at:

http://genesdev.cshlp.org/content/9/17/2193.full.html\#ref-list-1

License

Email Alerting

Service

Receive free email alerts when new articles cite this article - sign up in the box at the top right corner of the article or click here.

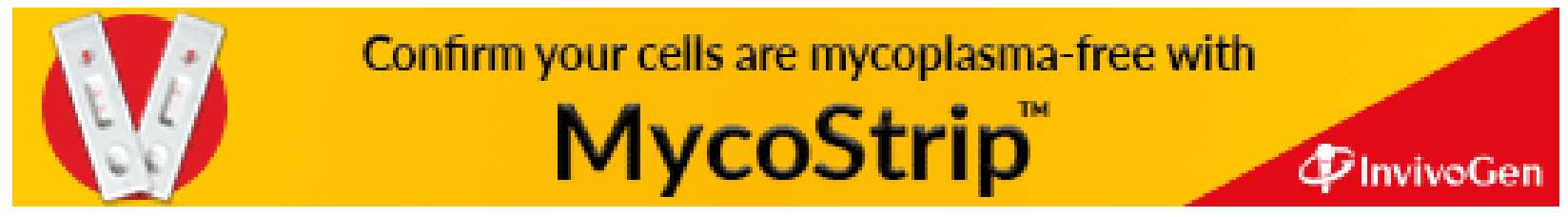

